\title{
Pharmakologische Beeinflussung des respiratorischen Stoffwechsels unter verschiedenen thermischen Bedingungen
}

\author{
WOLFGANG Usinger
}

\author{
W. G. Kerckboff-Institut der Max-Planck-Gesellschaft, Bad Nauheim
}

\begin{abstract}
Pharmacological effects on the respiratory metabolism under various thermal conditions. The only possible way to evaluate exactly pharmacological effects on the respiratory metabolism is to compare the results with the minimal rate, i. e., basal metabolic rate, in a thermo-indifferent environment. The initial values are not sufficient and may lead to wrong inferences. Experiments in a thermo-indifferent environment were carried out on dogs $\left(24^{\circ} \mathrm{C}\right)$ and mice $\left(30^{\circ} \mathrm{C}\right)$ in order to obtain their basal metabolic rates and to compare these rates with the effects of barbiturate narcosis, chlorpromazine and reserpine upon the oxygen consumption. There was no decrease in oxygen consumption below the basal metabolic rate in dogs or mice. Under the conditions of a non-thermo-indifferent environment $\left(0^{\circ} \mathrm{C}\right)$, oxygen consumption of dogs was reduced when barbiturate narcosis and chlorpromazine were applied; however, it did not sink beneath the basal metabolic rate measured at $24^{\circ} \mathrm{C}$, as thermoregulation was not blocked. In mice kept at $20^{\circ} \mathrm{C}$, on the other hand, the metabolic rate evidently decreased through the action of barbiturate narcosis and reserpine, resulting in the blocking of thermoregulation. The oxygen consumption under chlorpromazine also decreased, but no blocking of thermoregulation occurred.
\end{abstract}

\section{EINLEITUNG}

Die Bestimmung des respiratorischen Stoffwechsels bei pharmakologischen Untersuchungen hat in den vergangenen Jahren zu Ergebnissen geführt, die von physiologischer Seite nicht unwidersprochen geblieben sind (Literatur bei L'AlLEMAND et al. 1955, Brendel et a1. 1954, Thauer 1957, Usinger 1957a, b, 1962). Wenn man nämlich die Wirkung eines Pharmakons auf den respiratorischen Stoffwechsel beurteilen will, muß zum Vergleich der in Thermoindifferenz erhaltene Minimalwert des ruhenden, pharmakologisch unbeeinflußten Tieres herangezogen werden (BreNDel et al. 1954, Usinger 1957a). Es genügt nicht, die Wirkung mit dem sogenannten Ausgangswert zu vergleichen, der jeweils vor dem eigentlichen Versuch erhalten und von vielen Autoren als Ruhestoffwechsel angesehen wird, falls keine äußere Unruhe zu beobachten ist. Tatsächlich aber ist der Ausgangswert auch unter sogenannten Ruhe- oder Grundumsatzbedingungen großen Streuungen unterworfen, und der wahre Grundumsatz, d. h. der unter Beachtung bestimmter Kautelen in Thermoindifferenz erhaltene Minimalwert, wird nur in seltenen Fällen, manchmal sogar nur im Schlaf erreicht (Brendel et al. 1954, Usinger 1957a). 


\section{ERGEBNISSE}

Für Hunde ist ein unter Umständen monatelanges intensives Training erforderlich, um solche Minimalwerte zu erhalten (Methodik nach BrEndel et al. 1954). In Abbildung 1 sind auf der linken Seite als Beispiel die an zwei wohltrainierten Hunden erhaltenen Ergebnisse dargestellt, welche in 51 bzw. 26 Einzelversuchen in Thermoindifferenz bei $24^{\circ} \mathrm{C}$ erhalten wurden. Die Häufigkeitskurven zeigen, daß trotz äußerlicher Ruhe des Versuchstieres sein Sauerstoffverbrauch fast immer höher als der
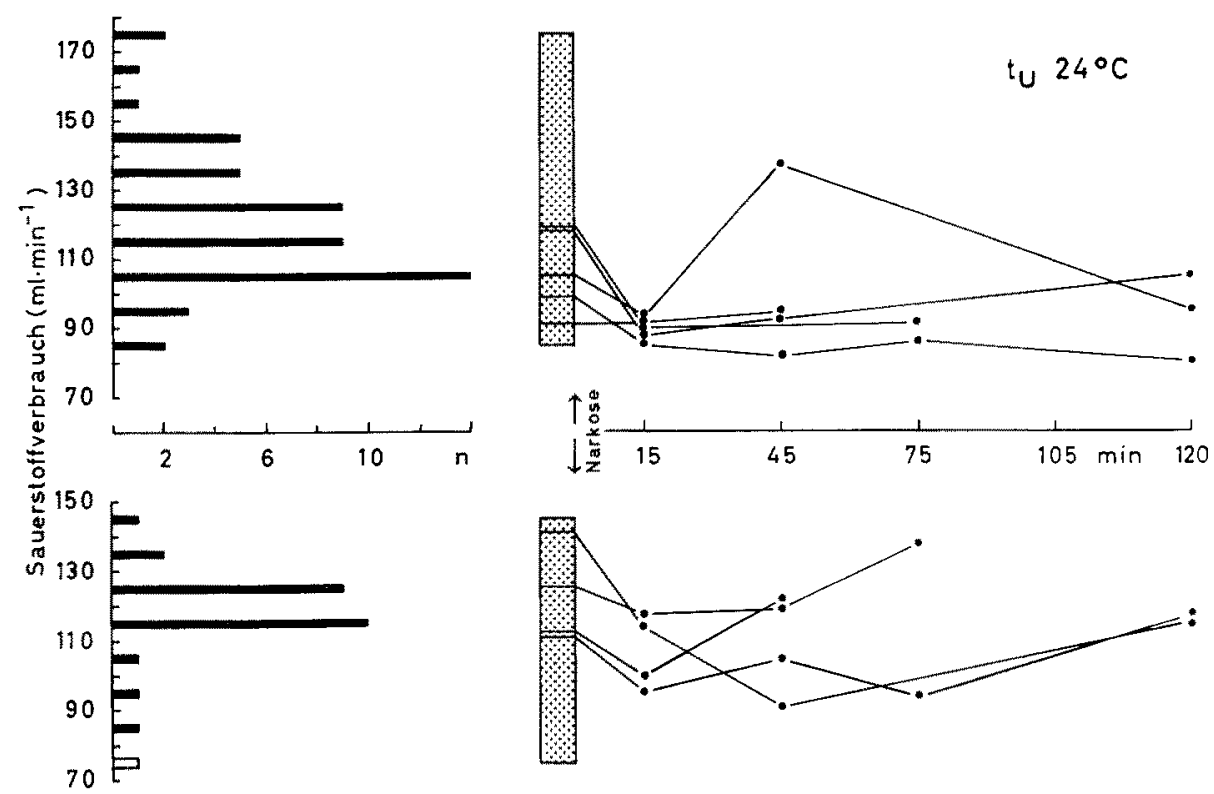

Abb. 1: Links: Verteilung der Ruhewerte des Sauerstoffverbrauches von 2 wohltrainierten Hunden in thermoindifferenter Umgebung $\left(24^{\circ} \mathrm{C}\right)$; offene Säule : Schlafwert. Rechts: Sauerstoff verbrauch der gleichen Hunde unter Barbituratnarkose im Vergleich zur Variationsbreite der Ausgangswerte (punktierte Säulen) in $24^{\circ} \mathrm{C}$ Umgebungstemperatur. (Keine Unterscheidung zwischen Evipan- oder Pernoctonnarkose, da lediglich die Narkosetiefe, nicht aber das Narkotikum, interessiert; siehe auch Abb. 3 und 5)

Minimalwert liegt und diesen, der identisch mit dem Schlafwert ist, nur in den wenigsten Fällen erreicht. Aus diesem Grunde ist das Verhalten des respiratorischen Stoffwechsels unter Barbituratnarkose verständlich (Abb. 1, rechte Seite). Führt man bei einem beliebigen Ausgangswert, der innerhalb der punktierten Sätlen liegen kann, eine Barbituratnarkose durch (Evipan $60 \mathrm{mg} / \mathrm{kg}$, Pernocton $50 \mathrm{mg} / \mathrm{kg}$, i. v.), so tritt, verglichen mit dem Ausgangswert, eine Senkung des Sauerstoffyerbrauches ein, die um so deutlicher ist, je höher der Ausgangswert liegt. Daraus könnte man den Schluß ziehen, daß eine spezifische Senkung des Sauerstoffverbrauches, im Sinne einer Histoplegie, vorliegen würde. Tatsächlich besagt dieses Ergebnis aber nichts weiter, als daß die tiefe Narkose den Sauerstoffverbrauch auf das Niveau des Grundumsatzes reduziert, oder anders ausgedrückt, daß der Minimalumsatz im Wachzustand mit dem in tiefer Narkose erreichbaren Minimalwert identisch ist. 
Da die Wirkung von Narkotika häufig an Kleintieren untersucht wird, wurde neben den Hundeversuchen eine entsprechende Serie an Mäusen durchgeführt. Die Maus setzt infolge ihrer Untrainierbarkeit der Bestimmung des Minimalstoffwechsels großen Widerstand entgegen, denn ihrer Verhaltensweise entsprechend, tritt nur für
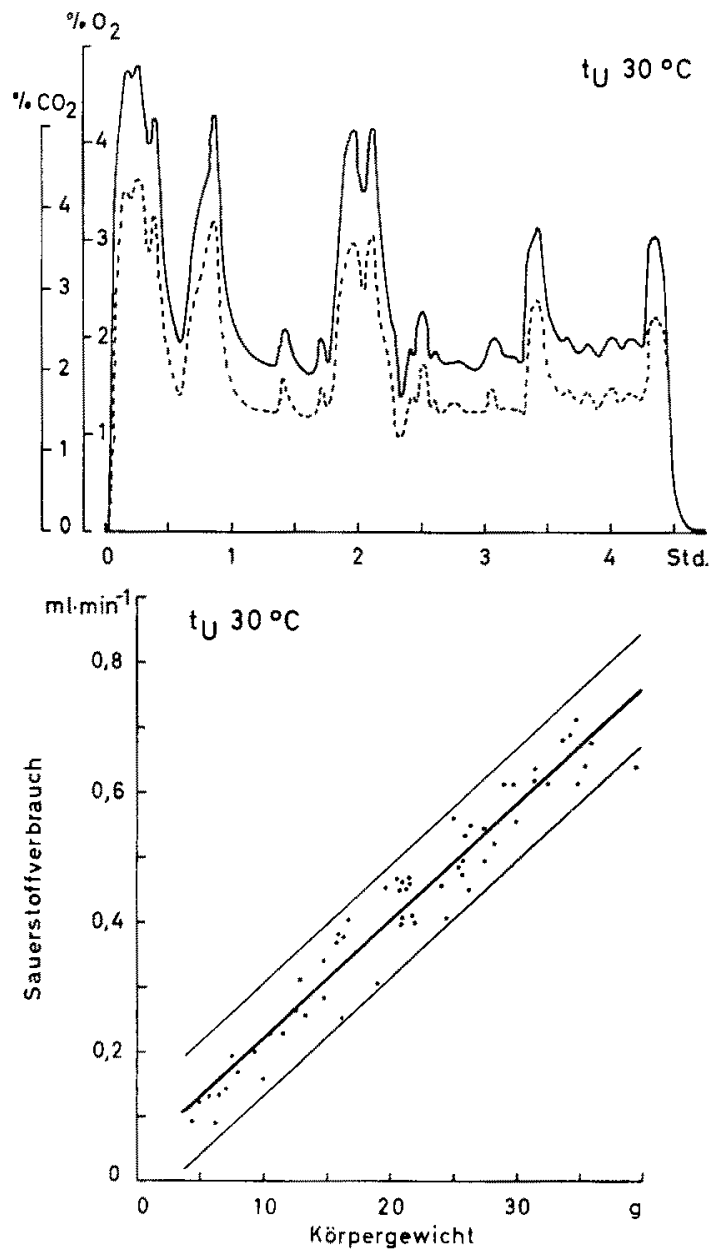

Abb. 2: Oben: Registrierte Originalkurve des respiratorischen Stoff wechsels einer weißen Maus in thermoindifferenter Umgebung $\left(30^{\circ} \mathrm{C}\right)$; ausgezogene Linie: Sauerstoffdefizit in $\%$; gestrichelte Linie: Kohlensäureiberschuß in $\%$. Unten: Beziehung zwischen minimalem Sauerstoffverbrauch und Körpergewicht von 60 weißen Mäusen in thermoindifferenter Umgebung $\left(30^{\circ} \mathrm{C}\right)$; dicke Linie: errechnete Regressionsgerade; dünne Linien: 2s-Grenzen

durchschnittlich 10 min ein Stoffwechselminimum ein, währenddessen sie ihre typische Schlafstellung einnimmt. Dieser Zustand ist praktisch nur mit fortlaufend registrierenden Methoden zu erfassen, da mit integrierenden Messungen das Stoff wechselminimum kaum ermittelt werden kann (UsINGer 1957a). In Abbildung 2 (oben) ist eine registrierte Originalkurve eines fünfstündigen Versuches wiedergegeben. Sie zeigt 
Perioden gesteigerter Stoffwechselaktivität, also Perioden gesteigerter Motilität und Ruheperioden, während der das Tier motorisch ruhig ist, aber nur ein einziges Mal das Stoffwechselminimum während des Schlafes. Bei der Maus sind Schlafperioden

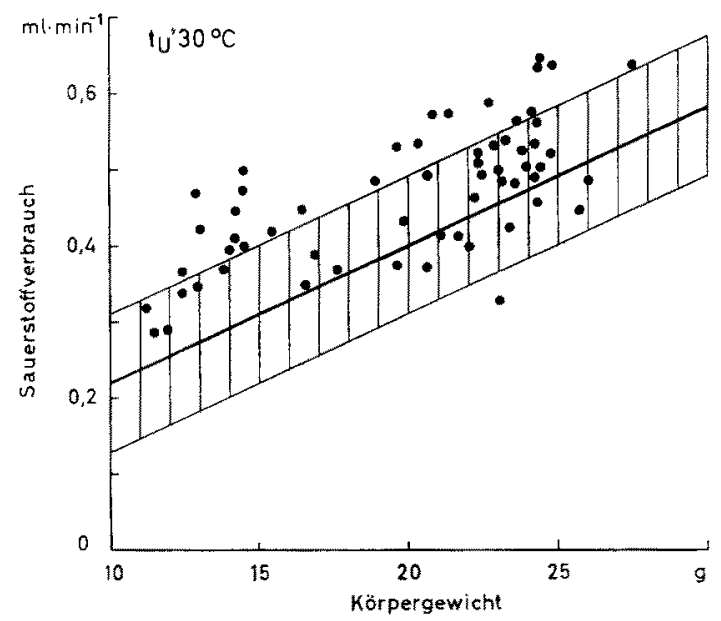

Abb. 3: Beziehung zwischen Sauerstoffverbrauch und Körpergewicht von 60 weißen Mäusen unter Barbituratnarkose. Vergleich zur Standardkurve normaler, unbeeinflußter Tiere. Versuche in thermoindifferenter Umgebung $\left(30^{\circ} \mathrm{C}\right)$
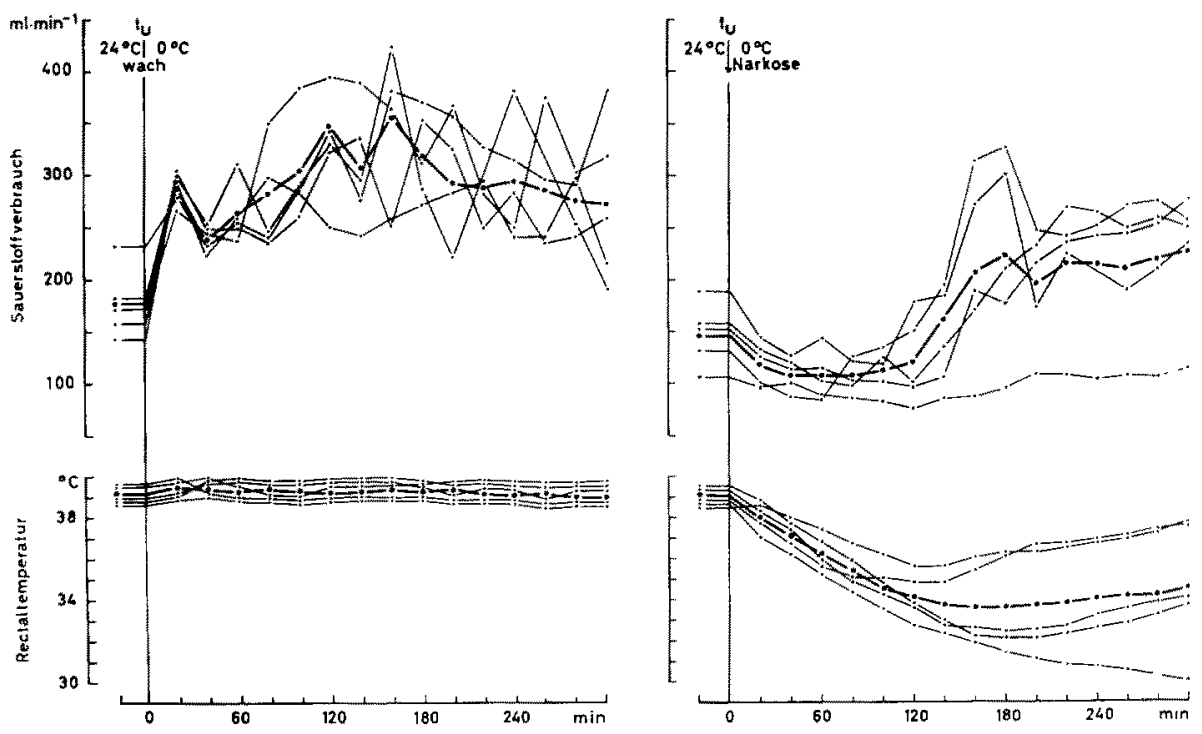

Abb. 4: Links: Sauerstoffverbrauch und Rectaltemperatur von 5 wachen Hunden in erniedrigter Umgebungstemperatur $\left(0^{\circ} \mathrm{C}\right)$. Rechts: Sauerstoffverbrauch und Rectaltemperatur der gleichen Hunde unter Barbituratnarkose (Pernocton) in $0^{\circ} \mathrm{C}$; dicke Linien: Mittelwertskurven

besonders durch die Atemfrequenz von motorischen Ruheperioden zu unterscheiden, die im ersteren Fall durchschnittlich 50 pro min beträgt (UsInger 1957a) und sehr rasch auf das Doppelte ansteigen kann, selbst wenn das Tier motorisch ruhig ist. Trägt 
man nun den jeweils tiefsten Wert des Sauerstoffverbrauches aus 60 derartigen Versuchen an 60 unterschiedlich schweren Tieten in die Parameter Sauerstoffverbrauch Körpergewicht ein (Abb. 2, unterer Teil), so ergibt sich eine gute Korrelation mit relativ geringer Streuung. Die errechnete Regressionsgerade $(Y=0,038+0,018 x)$ mit ihren 2s-Grenzen bildet die Standardkurve für die weiter vorzutragenden Ver-

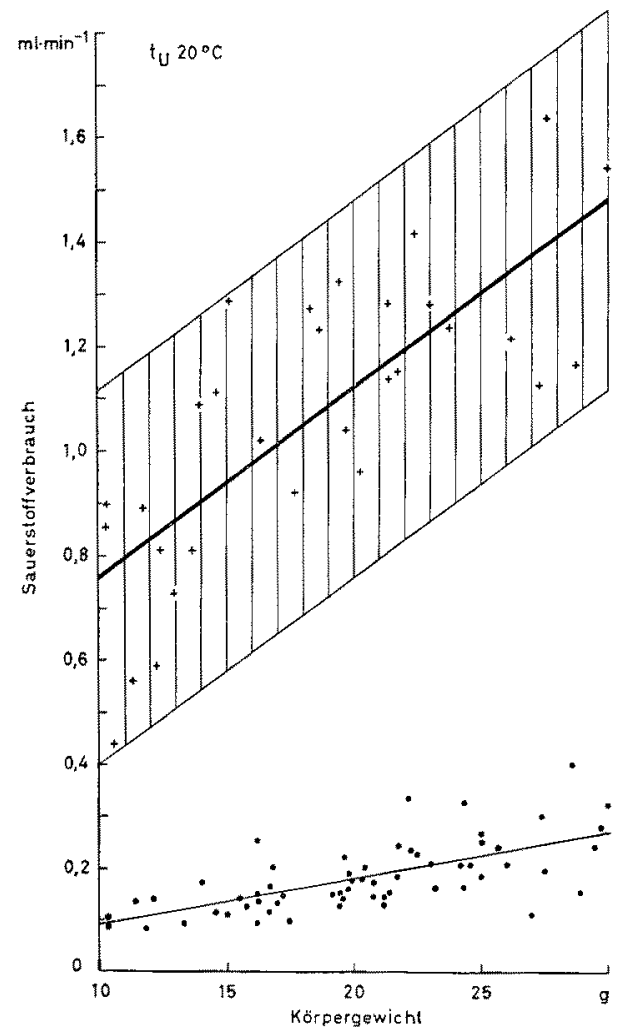

Abb. 5: Beziehung zwischen Sauerstoffverbrauch und Körpergewicht von weißen Mäusen in erniedrigter Umgebungstemperatur $\left(20^{\circ} \mathrm{C}\right)$, Kreuze und schraffiertes Band: Ruhewerte normaler, unbeeinflußter Tiere und die daraus errechnete Regressionsgerade mit 2s-Grenzen; Punkte und ausgezogene Linie: Minimalwerte unter Barbituratnarkose mit der errechneten Regressionsgeraden

suche. In Abbildung 3 sind die Minimalwerte aus 60 Einzelversuchen an 60 Tieren, die unter tiefer i. v. Barbituratnarkose erhalten wurden (Evipan $250 \mathrm{mg} / \mathrm{kg}$, Pernocton $125 \mathrm{mg} / \mathrm{kg}$ ), eingetragen, und man erkennt, daß nur mit einer Ausnahme, alle Werte im Streubereich der Standardkurve, oder sogar höher liegen. So läßt sich dar für den Hund Gesagte auch auf die Maus übertragen: Barbituratnarkose reduziert den respiratorischen Stoffwechsel, oder genauer gesagt, den Sauerstoffverbrauch auf das im unbeeinflußten Zustand erreichbare Stoffwechselminimum; keinesfalls unterschreiten die Narkosewerte den unter Grundumsatzbedingungen ermittelten Minimalwert.

Bei diesen Untersuchungen an Mäusen ist peinlich auf die Einhaltung der metabolischen Indifferenztemperatur von etwa $30^{\circ} \mathrm{C} z u$ achten, da sie im Gegensatz zum 
Hund wesentlich über der normalen Laboratoriumstemperatur liegt. Jegliche Anderung der Temperatur zu niederen und höheren Werten hin wird bei der Temperaturlabilität der Maus mit einer Steigerung des Sauerstoffverbrauches beantwortet und führt zu verfälschten Interpretationen, auf die noch einzugehen ist.

Wenden wir uns nun dem respiratorischen Stoffwechsel in niedriger Umgebungstemperatur zu. Derartige Untersuchungen werden immer dann durchgeführt, wenn man den Einfluß von Pharmaka auf die Kältegegenregulation prüfen

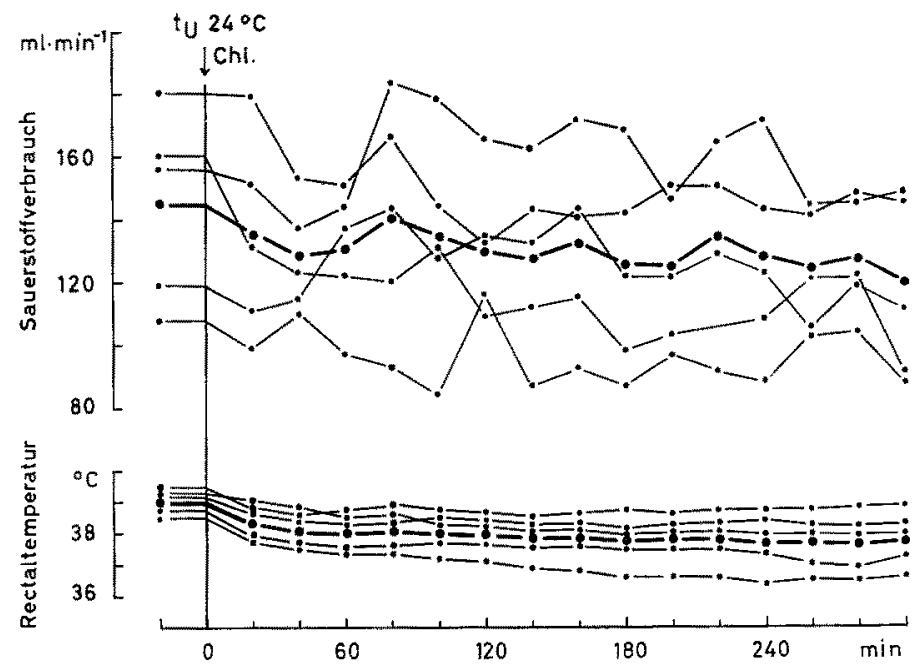

Abb. 6: Sauerstoffverbrauch und Rectaltemperatur von 5 Hunden unter der Wirkung von Chlorpromazin in thermoindifferenter Umgebung $\left(24^{\circ} \mathrm{C}\right)$; dicke Linien: Mittelwertskurven

will. Der normale, unbeeinflußte Organismus versucht in der Kälte durch gesteigerte Wärmeproduktion dem Wärmeentzug entgegenzuwirken, um ein Absinken der Körpertemperatur zu verhindern. Als Beispiel hierfür sind in der Abbildung 4 (linke Seite) die Ergebnisse von 5 Versuchen, die wir an 5 Hunden in $0^{\circ} \mathrm{C}$ Umgebungstemperatur durchgeführt haben, dargestellt. Sofort nach Uberführung in $0^{\circ} \mathrm{C}$ reagiert jeder Hund mit einem steilen Anstieg des Sauerstoffverbrauches, den er auch iiber Stunden beibehält, um seine Körpertemperatur auf gleicher Höhe zu halten. Wird bei diesen Hunden eine tiefe Barbituratnarkose (Pernocton) angewandt, wie es auf der rechten Seite der Abbildung $4 \mathrm{zu}$ erkennen ist, so wird die Kältegegenregulation stark gedämptt, d. h. der Wärmeentzug ist größer als die Wärmeproduktion. Daraus folgt ein Absinken der Körpertemperatur. Erst nach 2 Stunden setzt infolge der abklingenden Narkose die Gegenregulation wieder deutlich ein und ermöglicht durch den gesteigerten Sauerstoffverbrauch ein Wiederansteigen der Körpertemperatur. Vergleichen wir die Minima des Stoffwechsels in kalter Umgebung (Mittelwert $107 \mathrm{ml} \cdot \mathrm{min}^{-1}$ ) mit denen in Thermoindifferenz, die für diese Hunde im Mittel bei $75 \mathrm{ml} \cdot \mathrm{min}^{-1}$ liegen, so wird deutlich, daß trotz der reduzierten Körpertemperatur der Grundumsatz nicht unterschritten ist; mit anderen Worten müßte das heißen, daß selbst in tiefster Narkose beim Hund die Kältegegenregulation noch nicht erloschen ist. 
Versuche an der normalen, unbeeinflußten Maus in kalter Umgebung führten zu den prinzipiell gleichen, wenn auch quantitativ unterschiedlichen Ergebnissen: Während eines mehrstündigen Versuches in $20^{\circ} \mathrm{C}$ (was für die Maus bereits eine deutlich tiefere Umgebungstemperatur bedeutet) wechseln Perioden gesteigerter Stoffwechselaktivität mit Perioden motorischer Ruhe einander $a b$, ähnlich wie es die Versuche in

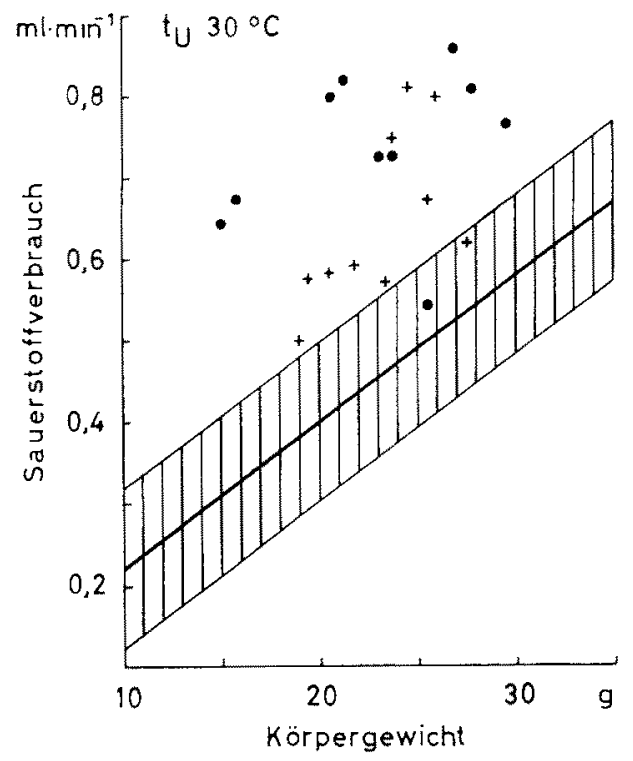

Abb. 7: Beziehung zwischen Sauerstoffverbrauch und Körpergewricht von weißen Mäusen unter der Wirkung von Chlorpromazin (Punkte) und Reserpin (Kreuze) in thermoindifferenter Umgebung $\left(30^{\circ} \mathrm{C}\right)$. Vergleich zur Standardkurve normaler, unbeeinflußter Tiere (schraffiertes Band)

Thermoindifferenz zeigen. Ein typischer Schlafzustand kommt nicht zustande, da die Maus bei $20^{\circ} \mathrm{C}$ eine sehr starke Gegenregulation aufweist. In der Abbildung 5 sind die Ruhewerte aus 30 derartigen Versuchen (Kreuze), also die tiefsten erreichbaren Werte eines Versuches, in das Koordinatensystem Sauerstoffverbrauch-Körpergewicht eingetragen und es ergibt sich wiederum eine lineare Abhängigkeit mit einer etwas größeren Streuung als in Thermoindifferenz (Regressionsgerade $\mathrm{Y}=0,406+0,036 \mathrm{x}$ ). Bei den Mäusen hat die Barbituratnarkose jedoch eine wesentlich tiefgreifendere Wirkung als beim Hund. Die Minimalwerte aus 60 Narkoseversuchen sind in der Abbildung 5 enthalten (Punkte) und liegen ca. 80\% unterhalb des Stoff wechsels unnarkotisierter Tiere. Die Narkose blockiert demnach die Kältegegenregulation vollständig, so daß die Körpertemperatur innerhalb von 2 Stunden auf Werte absinkt, die nur $1-2^{\circ} \mathrm{C}$ oberhalb der Umgebungstemperatur von $20^{\circ} \mathrm{C}$ liegen.

Wie aus den bis jetzt geschilderten Versuchen hervorgeht, beruht die häufig falsche Interpretation der Wirkung von Narkotica auf einer Nichtbeachtung der Unterschiede zwischen Ausgangswert und Minimalumsatz bzw. thermoindifferenter und nichtthermoindifferenter Umgebung. Angesichts dieser Erfahrungen interessierte es uns, auch die Wirkung solcher Sedativa zu untersuchen, denen eine spezielle histoplegische 
Tabelle 1

Maximalwerte des Sauerstoffverbrauches $\left(\mathrm{ml} \cdot \mathrm{min}^{-1}\right)$ von 5 Hunden unter verschiedenen Medikationen in erniedrigter Umgebungstemperatur $\left(0^{\circ} \mathrm{C}\right)$ während der ersten beiden Versuchsstunden

\begin{tabular}{|cccc|}
\hline Hund & wad & Chlorpromazin & Pernocton \\
\hline 1 & 345 & 218 & 144 \\
2 & 322 & 321 & 124 \\
3 & 330 & 223 & 101 \\
4 & 394 & 311 & 119 \\
5 & 299 & 294 & 150 \\
\hline
\end{tabular}

Wirkung, d. h. eine Senkung des Sauerstoffverbrauches unter den Grundumsatz, zugesprochen wurde, wie z. B. dem Chlorpromazin und Reserpin.

Betrachten wir zunächst die Wirkung von Chlorpromazin $(10 \mathrm{mg} / \mathrm{kg} \mathrm{i.} \mathrm{v.)} \mathrm{beim}$ Hund in thermoindifferenter Umgebung. Abbildung 6 zeigt, daß im Verlauf von mehreren Stunden eine leichte Senkung des Sauerstoffverbrauches gegenüber dem Ausgangswert eintritt, ebenso ein geringfügiger Abfall der Rectaltemperatur. Doch

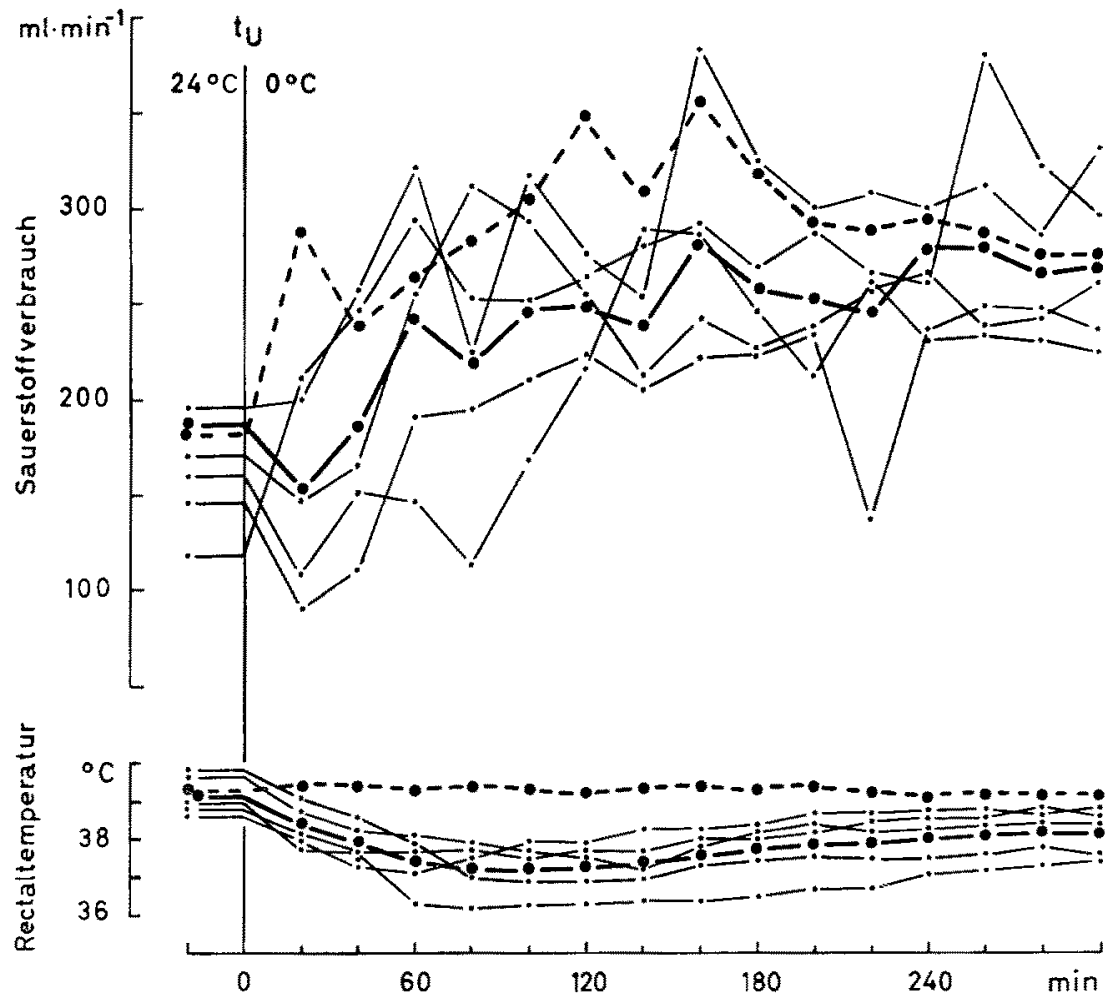

Abb. 8: Sauerstoffverbrauch und Rectaltemperatur von 5 Hunden unter der Wirkung von Chlorpromazin in erniedrigter Umgebungstemperatur $\left(0^{\circ} \mathrm{C}\right)$; dünne Linien: Einzelversuche; dicke Linien: Mittelwertskurven; dicke gestrichelte Linien: Mittelwertskurven der normalen, unbeeinflußten Kontrolltiere (siehe auch Abb. 4, links) 
wird auch hier der Minimalumsatz der Hunde, der uns aus vorangegangenen Versuchen bekannt war, nicht unterschritten.

Bei der Maus sind die Verhältnisse unter Chlorpromazin $(10 \mathrm{mg} / \mathrm{kg} \mathrm{i.} \mathrm{v}$ ) aus anderen Gründen etwas komplizierter. Aus Abbildung 7 geht hervor, daß die tiefsten Werte unter Chlorpromazin (Punkte) deutlich höher als die Minimalwerte liegen (Standardkurve), weil Chlorpromazin die Wärmeabgabe erhöht und deshalb die Indifferenztemperatur zu höheren Werten verschoben ist. Ein ähnliches Bild geben die Versuche mit Reserpin wieder ( $1 \mathrm{mg} / \mathrm{kg}$ i. v.), die in der Abbildung 7 als Kreuze eingetragen sind. Für eine histoplegische Wirkung dieser beiden Pharmaka ist demnach auch bei den Mäuseversuchen kein Anhaltspunkt zu finden.

Wenden wir uns den Ergebnissen der Chlorpromazin- und Reserpinversuche in niedriger Umgebungstemperatur zu, finden wir folgende Resultate: In $0^{\circ} \mathrm{C}$ wird beim Hund durch Chlorpromazin die Kältegegenregulation nicht blockiert, sondern nur geringgradig abgeschwächt, ebenso fällt die Rectaltemperatur nur um wenige Grade ab. In der Abbildung 8 ist die Mittelwertskurve unbeeinflußter Tiere im Vergleich zu der mit Chlorpromazin behandelten dargestellt, ebenso die entsprechenden Rectaltemperaturen. Um eine Vorstellung über die Intensität der Kältegegenregulation normaler und mit Chlorpromazin behandelter Tiere zu erhalten, sind in Tabelle 1 die jeweiligen Maximalwerte des Sauerstoffverbrauches von 5 Hunden, die während der ersten beiden Versuchsstunden gewonnen wurden, vergleichsweise zusammengestellt.

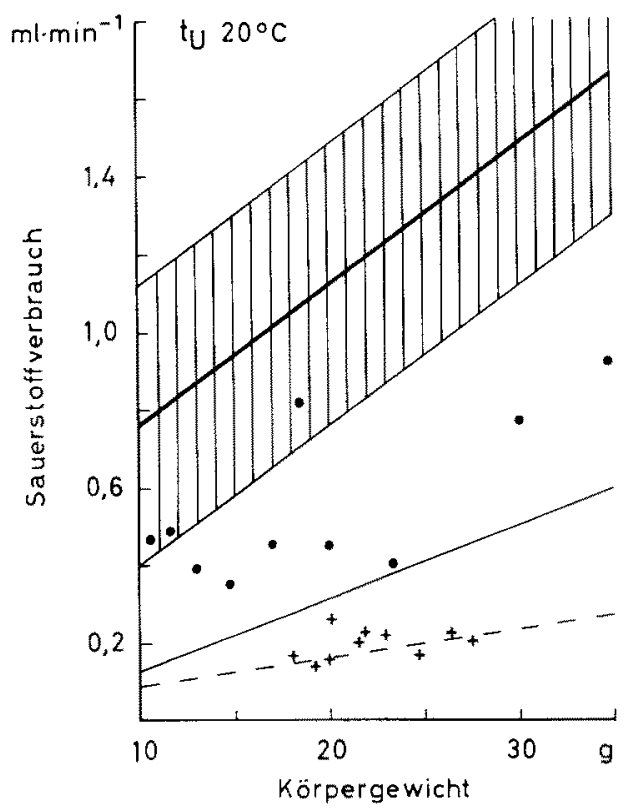

Abb. 9: Beziehung zwischen Sauerstoffverbrauch und Körpergewicht von weißen Mäusen unter der Wirkung von Chlorpromazin (Punkte) und Reserpin (Kreuze) in erniedrigter Umgebungstemperatur $\left(20^{\circ} \mathrm{C}\right)$. Gestrichelte Linie: Regressionsgerade der Werte unter Barbituratnarkose (nach Abb. 5); ausgezogene Linie: untere $2 s$-Grenze der Standardkurve in Thermoindifferenz von $30^{\circ} \mathrm{C}$ (nach Abb. 2); schraffiertes Band: Standardkurve normaler, unbeeinflußter T'iere in $20^{\circ} \mathrm{C}$ Umgebungstemperatur (nadh Abb. 5) 
Aus diesen Werten geht hervor, daß durch Chlorpromazin nur eine geringfügige Dämpfung des Sauerstoffverbrauches gegenüber den normalen, unbeeinflußten Tieren auftritt, daß aber durch die Narkose eine drastische Reduktion verursacht wird.

Bei der Maus hat das Chlorpromazin in $20^{\circ} \mathrm{C}$ Umgebungstemperatur ebenfalls nur eine stoffwechselreduzierende Wirkung, wenn auch die Rectaltemperatur einen wesentlich stärkeren Abfall um etwa $10^{\circ} \mathrm{C}$ aufweist. Aus Abbildung 9 ist zu ersehen, daß selbst die Minimalwerte des Sauerstoffverbrauches unter Chlorpromazin (Punkte) immer noch im unteren Streubereich der unter thermoindifferenten Bedingungen gewonnenen Standardkurve liegen, deren untere 2s-Grenze die ausgezogene Linie darstellt. Die Wirkung von Reserpin hingegen (Kreuze) ist wesentlich stärker und kann mit der Narkosewirkung (deren Regressionsgrade: gestrichelte Linie) verglichen werden. Hier ist die Kältegegenregulation vollständig erloschen.

\section{ZUSAMMENFASSUNG}

1. Selbst unter Einhaltung aller Grundumsatzbedingungen, auf die BENEDICT in seinem Buche „Vital Energetics" (1938) hingewiesen hat, wird sowohl bei großen als auch bei kleinen Tieren nur selten der Minimalumsatz erreicht. Um ihn zu ermitteln, bedarf es eines langfristigen Trainings bzw. einer besonderen Methodik.

2. Die untersuchten Pharmaka aus der Reihe der Barbiturate und Sedativa vermögen weder bei großen noch bei kleinen Warmblütern den respiratorischen Stoffwechsel unter den Minimalumsatz zu senken, wenn die Versuche in thermoindifferenter Umgebung durchgeführt werden.

3. Die gleichen Pharmaka bewirken in tieferen Umgebungstemperaturen eine quantitativ verschiedene Einschränkung der Kältegegenregulation. Dabei zeigen sich die Barbiturate und das Reserpin dem Chlorpromazin weit überlegen.

4. Unterschiede im Verhalten großer und kleiner Tiere in kalter Umgebung sind dadurch zu erklären, daß bei den Kleintieren eine Blockierung der Gegenregulation zu stärkerer Auskühlung, d. h. tieferen Senkung der Körpertemperatur, führt.

5. Versuche über die Wirkung sedativer Pharmaka auf die Temperaturregulation sollten deshalb bei Kleintieren durchgeführt werden, da bei ihnen schon geringste Störungen in dem Gleichgewicht Wärmeproduktion-Wärmeabgabe große meßbare Unterschiede hervorrufen.

\section{ZITIERTE LITERATUR}

L'Allemand, H., Brendel, W. \& Usinger, W., 1955. Über den Mechanismus der Chlorpromazin-(Megaphen-) Wirkung auf die Temperaturregulation. Anaesthesist 4, 36-41.

BeNFDict, F. G., 1938. Vital Energetics. A study in comparative basal metabolism. Carnegie Institution Publication No. 503, Washington, D. C., 215 pp.

Brendex, W., Koppermann, E. \& Thauer, R., 1954. Der respiratorische Stoffwechsel in Narkose. (Ein Beitrag zur Frage des Minimalumsatzes.) Pfingers Arch. ges. Physiol. 259, $177-206$.

Thauer, R., 1957. Kreislauf in Narkose. Verh. dtsch. Ges, Kreislaufforsch. 23, 1-33. 
UsINGER, W., 1957a. Respiratorischer Stoff wechsel und Körpertemperatur der weißen Maus in thermoindifferenter Umgebung. Pflügers Arch. ges. Physiol. 264, 520-535.

- 1957b. Respiratorischer Stoffwechsel und Körpertemperatur der weißen Maus in Narkose und unter Chlorpromazin. Pflügers Arch. ges. Physiol. 265, 365-381.

- 1962. Die Wirkung von Reserpin auf die Temperaturregulation. Arzneim.-Forsch. 12, $435-438$.

\section{Diskussion im Anschluß an den Vortrag UsINGer}

Heusner: Bei ganz ähnlichen Versuchen an der Ratte habe ich ein starkes Absinken der Körpertemperatur und des Energiewechsels in Barbituratnarkose festgestellt, so daß der Energiewechsel weit unter dem normalen Grundumsatz der Ratte liegt.

USINGER: Zu den Ergebnissen an Ratten kann keine Stellung genommen werden, da keine eigenen Untersudhungen vorliegen, jedoch scheint es, daß der sogenannte Grundumsatz nicht dem tatsächlichen Minimalumsatz entspricht.

OHNesorge: Welche Dosierung von Chlorpromazin und Reserpin wurde bei den Mäusen verwendet? $\mathrm{Zu}$ welchem Zeitpunkt wurde Reserpin gegeben?

Usinger: Chlorpromazin wurde in den vorgetragenen Versuchen in einer Dosierung von $10 \mathrm{mg} / \mathrm{kg}$ i. v. gegeben; Reserpin, $1 \mathrm{mg} / \mathrm{kg}$ i. v., wurde zu Versuchsbeginn injiziert.

SMITH: The figures showing the relation of oxygen consumption to body weight for normal and narcotized animals actually display equal slopes, but these do not appear equal because of the arithmetic coordinates employed.

UsINGER: Die Versuchsergebnisse wurden nicht in dieser Richtung ausgewertet. 